\title{
LA CREACIÓN LÉXICA MEDIANTE SIGLAS: UN RECURSO DE CONDENSACIÓN EXPRESIVA EN LA PRENSA DIGITAL.
}

\author{
Carlos Oliva-Marañón ${ }^{1}$ Universidad Rey Juan Carlos. España. \\ carlos.oliva.maranon@urjc.es
}

\section{RESUMEN}

Las siglas en la Lengua Española parecían tener menor vitalidad si se comparaban con las lenguas de otros países. Sin embargo, han cobrado un considerable protagonismo en las últimas décadas, de tal forma que algunos lexicógrafos han considerado a España como el país "abreviaturóforo" debido especialmente, al desarrollo de este subsistema léxico por la proliferación de organizaciones políticas que surgen a partir del año 1975. Puede decirse que una sigla se halla tanto mejor integrada como elemento del léxico, cuanto más borrada está su motivación original. En el aspecto morfosintáctico, la referencia a organizaciones e instituciones de variado orden determina el carácter sustantivo de las siglas, la mayoría de las cuales constituyen nombres propios. El ingente caudal de voces sigladas y el uso tan frecuente de éstas tanto en la prensa tradicional como en la digital, ejercen una presión estimable en el sistema lingüístico. La mayoría de las siglas en español se ajustan al sistema fonológico, pero también son numerosas las que se apartan de él al adoptar algunas de sus vocales y consonantes una distribución ajena al sistema lingüístico. El carácter nominal puede darse en siglas que no han producido derivados, lo mismo que ocurre con las palabras tradicionales.

PALABRAS CLAVE: Siglas - Léxico - Prensa digital - Sistema lingüístico - Fenómeno lexicológico.

\footnotetext{
${ }^{1}$ Autor correspondiente

Carlos Oliva-Marañón: Profesor Doctor. de la Facultad de Ciencias de la Comunicación, Universidad Rey Juan Carlos., España.

Correo: carlos.oliva.maranon@urjc.es
} 


\title{
THE LEXICAL CREATION BY MEANS OF INITIALS: A RESOURCE OF EXPRESSIVE CONDENSATION IN THE DIGITAL PRESS.
}

\begin{abstract}
IThe initials in the Spanish language would seem to have minor vitality if it is compared with the languages of other countries. Nevertheless, they have received a considerable protagonism in the last decades, in such a way that some lexicographers have considered Spain to be the country "abreviaturóforo" owed specially, to the development of this lexical subsystem for the proliferation of political organizations that arise from the year 1975. It can be said that an abbreviation is better integrated so much as element of the lexicon, more erased his original motivation is. In the aspect morphology- syntactic, the reference to organizations and institutions of varied order determines the substantive character of the initials, the majority of which constitute own names. The enormous flow of voices siglated and such a frequent use of these both in the traditional press and in the foxglove, they exercise an estimable pressure in the linguistic system. The majority of the initials in Spanish adjust to the phonological system, but also they are numerous those who are separated of him when adopt some of his members and consonants a distribution foreign to the linguistic system. The nominal character can be given in initials that have not produced derivatives the same as it happens with to traditional words.
\end{abstract}

KEY WORDS: Initials - Lexicon - Digital press - Linguistic system - Phenomenon lexicology.

\section{INTRODUCCIÓN}

El escritor español Pedro Salinas acuñó la frase de "el siglo de las siglas" con clara referencia al siglo XX. Sin embargo, en la actualidad, este fenómeno lexicológico sigue patente en el léxico castellano, sin gran diferencia de lo que está ocurriendo en todas las lenguas de cultura. Se llama sigla tanto a la palabra formada por las iniciales de los términos que integran una denominación compleja, como a cada una de esas letras iniciales. Las siglas se utilizan para referirse de forma abreviada a organismos, instituciones, empresas, objetos, sistemas, asociaciones, etc.

Debido a sus particularidades grafemáticas, las siglas se han venido considerando como una fuente de formación de léxico, si bien fuente marginal, relegada a la periferia del idioma, por lo que casi nunca han sido incluidas en las descripciones lingüísticas. Cuando el procedimiento se ha extendido, el carácter un tanto súbito de su aparición unido a su especificidad léxica ha llevado a calificar esta avalancha de términos como "plaga" o "manía" y a darles un tratamiento meramente superficial. 
El literato Dámaso Alonso rechazaba a la sigla, a la que denominaba "gris ejército esquelético", "legión de monstruos" o "fríos andamiajes"2. Igualmente, Rafael Lapesa, filólogo y miembro de la Real Academia Española de la Lengua, consideraba a la sigla como "plaga universal de monstruos que, por no tener raíz léxica, no evocan nada, y cuyo cuerpo sonoro, formado por azar y no por evolución orgánica, se eriza de combinaciones fonéticas inusitadas para nuestros oídos"3 (Lapesa, 1963, p. 201).

También, en este sentido, Manuel Casado Velarde nos describe en su obra Tendencias en el léxico español, cómo las siglas han invadido el vocabulario actual con aceleración creciente, de tal manera que apenas hay una esfera de él que no haya capitulado al empuje poderoso de este fenómeno. Cita el autor cómo se cuenta por centenares el número de siglas en cualquier periódico o revista de actualidad. Otro tanto sucede en muchos tratados técnicos, científicos o humanísticos. Y hasta en el lenguaje coloquial más íntimo cobran vida fonética estos elementos filológicos, no sólo en niveles lingüísticos cultos, sino también en los semicultos y aún en los vulgares. Este fenómeno lingüístico se circunscribe igualmente a la prensa digital, ya que el auge de las telecomunicaciones en la denominada Sociedad de la Información, también conocida como Sociedad Red, ha producido una profunda transformación de las Tecnologías de la Información y de la Comunicación.

\section{DESARROLLO}

\subsection{Definiciones lexicológicas de las siglas}

Acerca del lenguaje, el filólogo José Carlos Martín nos plantea la siguiente dicotomía: “La labor de categorización no la realizan del mismo modo el lenguaje común y el científico: mientras que el común se guía por la intuición y por patrones culturales, el científico se fundamenta en una objetividad con la que aspira a eliminar cualquier posible equívoco o ambigüedad. Esto significa, al menos en teoría, que las palabras comunes proporcionan interpretaciones "sesgadas" de las cosas y las científicas, en cambio, imágenes fieles de ellas, ya que su contenido se establece por parámetros basados en la precisión y el rigor" 4 (Martín Camacho, 2008).

Con referencia a las siglas, existen diferentes corrientes conceptuales relativas a su definición. En este sentido, se han expuesto diversos análisis que presentan importantes divergencias entre sí, aunque todas ellas se deben a una sola razón: la consideración de cuáles son los segmentos que actúan en la creación de la nueva unidad léxica.

2 Alonso, D. (1962) La invasión de las siglas. En: Del Siglo de Oro a este siglo de siglas, p. 7.

${ }^{3}$ Lapesa, R. (1963) La lengua desde hace cuarenta años. En: Revista de Occidente, núm. 8-9, p. 201.

${ }^{4}$ Martín Camacho, J. C. (2008) Sinonimia y polisemia en el léxico científico. El caso de las abreviaturas, las siglas y los epónimos. En: Olza, I, Casado, M. \& González, R. (ed.) Actas del XXXVII Simposio Internacional de la Sociedad Española de Lingüística (SEL). Pamplona: Servicio de Publicaciones de la Universidad de Navarra. 
Algunos autores sostienen, implícita o explícitamente, que la sigla se forma sólo con la primera letra de cada una de las palabras componentes del sintagma originario (cfr. por ej. Rodríguez González, 1993, p. 10; Alba de Diego ${ }^{5}$, 1973, p. 373; Casado Velarde, 1979, pp. 71-72; Miranda, 1994, pp. 168-172. En tal caso, no suelen establecerse tipologías, ya que sólo se toman en consideración formas como PSOE, PP o bien RENFE, que se explica como una excepción en la que se recurre a más letras que la inicial para formar un resultado que pueda actualizarse secuencialmente.

Otros estudiosos, en cambio, consideran que la sigla puede construirse no sólo con las letras iniciales de cada una de las palabras que forman la base, sino también con secuencias de varios fonemas (vid. Por ej. Alvar Ezquerra, 1983, p. 7ss. y 1996, pp. 4648; Almela, 1999, p 210 ss.; Lang, 1997, pp. 255-258; Figueroa, 1969). De este modo, se incluyen en las siglas formaciones como las anteriormente citadas, pero también otras de apariencia bastante distinta, caso de HUNOSA (Hulleras del Norte, S.A.), COFARMA (Comercial Farmacéutica Madrileña) o POLISARIO ("Frente" Popular para la Liberación de Sakiet el Hamra y Río de Oro). A partir de ahí, se distinguen diversos tipos de siglas. Otro vocablo importante es el término "sigloide", definido por A. Rosell como "siglas aparentes en las que se han utilizado términos secundarios, omitido fundamentalmente, o no se ha respetado el principio primario de tomar de los vocablos sólo la letra inicial" 6 .

Por su parte, Figueroa establece una interesante tipología en la que, sin emplear denominaciones específicas, distingue tres clases de siglas ${ }^{7}$ (Figueroa, 1969, pp. 6787): las formadas por el primer grafema de cada una de las palabras de base; las formadas por segmentos silábicos (normalmente, pero no de forma exclusiva, las iniciales) de las palabras del sintagma originario; y aquellas que muestran una segmentación arbitraria de las palabras originarias, ya que incluyen grafemas, sílabas y fragmentos no silábicos combinados de muy diversas maneras.

Por tanto, hay que establecer una diferenciación entre los términos abreviatura, sigla y sigloide. Con el término abreviatura, se designa el modo exclusivamente gráfico de representar las palabras con una o varias de sus letras: ejemplos, las grafías de Sr. D. que habitualmente preceden al nombre en las cartas y escritos de carácter administrativo; Dr. de Doctor, Km. de Kilómetro, etc. Lo característico de estos acortamientos es que no trascienden al plano oral. Su lectura restablece lo omitido en la representación gráfica.

\footnotetext{
${ }^{5}$ Según Alba de Diego, cuando la abreviación es grafémica suele denominarse sigla. Por su parte, en el diccionario de Galende (2000:9) parece establecerse una distinción entre sigla (formada por un solo grafema de cada una de las palabras del sintagma originario) y acrónimo (formado por más de un grafema), propuesta que complica aún más el panorama terminológico.

${ }^{6}$ Rosell, A. (1967) Escritura de formas siglares. Montevideo: La Paz, p. 34. Este autor emplea además el término seudosigla, que define como "cualquier forma léxica aparentemente siglar, compuesta caprichosamente".

${ }^{7}$ Figueroa, L. (1969) Algunas observaciones sobre siglas usuales en Bogotá. En: Thesaurus. BICC, XXIV, pp. 66-91.
} 
En cuanto al término sigla, las dos primeras acepciones que recoge el Diccionario de la Real Academia Española de la Lengua (DRAE) son:

a) "Letra inicial que se emplea como abreviatura de una palabra".

b) "Rótulo o denominación que se forma con varias siglas".

Así pues, en opinión del filólogo Manuel Casado Velarde, "lo que hay que destacar de esta definición académica es que para hablar de sigla en stricto sensu, se precisa que aparezca sólo el primer grafema de cada uno de los componentes del sintagma no abreviado, excepción hecha de los elementos de relación (preposiciones y conjunciones)" 8 (Casado Velarde, 1979, pp. 67-88). Y añade: “Así definida, la extensión significativa de la voz sigla queda enormemente reducida. El adjetivo "síglico", aunque no se incluye en el diccionario académico, es derivado muy frecuente en la bibliografía sobre el tema" 9 (Casado Velarde, 1979, p. 71).

Mediante la sigla se asegura la presencia de cada uno de los constituyentes de una determinada unidad sintagmática en una nueva secuencia lingüísticamente más económica. Una doble tensión está presente en la creación de una sigla, según L. Guilbert: de una parte, "el deseo de conseguir una reducción gráfica y fonética de la secuencia sintáctica, considerada demasiado larga para ser fácilmente utilizable en la comunicación; de otra, el cuidado por mantener la relación sintáctica entre los elementos, mediante la referencia a cada componente del conjunto" 10 (Guilbert, 1975, p. 275).

En la sigla se asiste a una inversión de las relaciones entre lengua hablada y lengua escrita. Los grafemas que la componen, al independizarse de la unidad de que formaban parte, adquieren autonomía; y unas nuevas relaciones, "sintagmáticas" se entablan entre grafemas desgajados, hasta el punto de adquirir en ocasiones nuevos valores fonológicos. Ocurre así, por ejemplo, en CEDA, donde el grafema $C$ ha cambiado su valor fonético (k) de Conferederación por el de (o), exigido, en la lectura integrada de las siglas, por un nuevo contexto gráfico. Lo oral va, aquí a la zaga de lo gráfico.

\subsection{Promptuario histórico de las siglas}

Respecto a la antigüedad de las siglas, el filólogo Rafael Seco nos recuerda cómo en su sentido primigenio se remonta, cuando menos, al tiempo de los romanos. En sentido moderno, su antigüedad no rebasa más allá de cien años, aunque, sin duda, sus comienzos debieron de ser tímidos, hacia finales del siglo pasado. De lo que no

\footnotetext{
87 Casado Velarde, M. (1979). Creación léxica mediante siglas. En: Revista Española de Lingüística, núm 9, Vol. 1, pp. 67-88.

${ }^{9}$ Ídem. p. 71.

${ }^{10}$ Guilbert, L. (1975) La créativité lexicale. París: Larousse, p. 275.
} 
parece caber duda es de que el uso masivo de las siglas modernas en los medios de comunicación impresos (especialmente, en los periódicos y, más particularmente, en los diarios) data de los años treinta. A partir de entonces, el uso de la sigla ha aumentado enormemente, hasta el punto de que no existe publicación actual en la que no aparezcan varios ejemplos ${ }^{11}$ (Seco, 1988, p. 191).

La postura ante la sigla ha variado mucho desde que se inició su frecuencia masiva. En los años treinta, Don Santiago Ramón y Cajal decía que, para aclarar su significado, haría falta, en frase de un ministro, "crear una asignatura universitaria". En su opinión, las siglas eran una importación del extranjero. Y citaba: “Carecemos de originalidad hasta para lo frívolo" 12 . Transcribimos su denuncia realizada en 1934 sobre el creciente uso de las siglas y que no puede tener más actualidad:

“...de la manía de abreviar los apelativos no se libran los partidos, ni las corporaciones, ni las pandillas deportivas. Y ha surgido una enigmática sarta de charadas, para aclarar las cuales haría falta, según frase de un ministro, crear una asignatura universitaria. Citemos tan sólo algunas de estas denominaciones misteriosas: U.G.T., F.A.I., C.N.T., (...) Y por no molestar prescindimos de las innumerables iniciales con que se designan las sociedades deportivas. Mencionarlas todas sería cuento de nunca acabar. Tales novelerías, según dejamos dicho, son importaciones extranjeras". ("El mundo a los ochenta años", en Obras literarias completas. Madrid, 1961'13, p. 329).

En 1967, Rosenblat, más pragmático, reconoce "el triunfo universal de las siglas" debido a la tendencia "a la palabra más corta, y a los modos expresivos más condensados"14 (Rosenblat, 1967, p. 164). Igualmente, Gerardo Diego afirmaba:

“Tal como van las cosas, dentro de no mucho tiempo, cuando ya todo el vocabulario se haya vuelto siglas, habrá que pensar en sacar las siglas de las siglas, las siglas de segundo grado hasta llegar a la pura álgebra y a la más desolada y electrónica abstracción"15 (Diego, 1968, p. 40).

A las siglas se recurre, por ejemplo, para denominar a varios organismos de alcance internacional: ONU, UNESCO, OTAN, UE, etc. En un ámbito más restringido, las siglas aparecen para designar no sólo los numerosos partidos políticos, entidades públicas y privadas, casas o marcas comerciales, sino también realidades tan familiares como son, en el campo de la enseñanza, los niveles educativos, instituciones, y títulos, como: EGB, BUP, COU, FP, CAP, INEM, INB, UNED, EEES, ECTS, PIO, INAPE.

\footnotetext{
11 Seco, R. (1988). Manual de Gramática Española. Madrid: Aguilar, p. 191.

12 Ramón y Cajal, tomado de Diego, G. (1968) Por las siglas de las siglas. En La Gaceta Literaria, p. 40.

${ }^{13}$ Ídem. p. 329.

14 Diego, G. (1968) Por las siglas de las siglas. La Gaceta Literaria, p. 40.

15 Rosenblat, A. (1967). El futuro de la lengua. En Revista de Occidente, núm. 56-57, p. 164.
} 
El mundo de la milicia ofrece, por su parte, designaciones del tipo de CIR, IMEC, IMECAR, las antiguas IPS y MAU, así como otras de carácter más especializado. Finalmente, ya en el reducto que podría llamarse doméstico, el menos proclive espontáneamente, en principio, a este fenómeno léxico, se usan con frecuencia formaciones síglicas que la mayoría de sus usuarios no sabe desarrollar: LP, TER, TALGO, SIDA, OVNI, SER, DDT, KO..., como únicas denominaciones conocidas de los respectivos referentes, que les vienen impuestas con ellos. También, Manuel Seco citaba que "esta exposición de siglas es un fenómeno que reclama, por lo que al castellano se refiere, la atención del lingüista, especialmente, la del lexicólogo" 16 (Seco, 1977, p. 193).

En este sentido, la Fundación del Español Urgente ${ }^{17}$ (Fundéu), que tiene como objetivo colaborar en el uso correcto del español en los medios de comunicación, recomienda usar, por ejemplo, la palabra "pyme" como un nombre común y no como una sigla. Tampoco es correcto escribir esa palabra entre comillas "pyme", pues, al estar ya incluida en el Diccionario de la Real Academia Española (el que la describe como un acrónimo de pequeña y mediana empresa, esto es, una empresa mercantil, industrial, etc., compuesta por un número reducido de trabajadores y con un moderado volumen de facturación), debe considerarse como una palabra más a todos los efectos. Así, no parece extraño ni incorrecto que de la palabra "pyme" se forme el plural regular "las pymes".

A veces, ciertas siglas siguen un proceso de lexicalización que las lleva a transformarse en nombres comunes: "GEO", "OPA", etc. Las palabras que se han formado a partir de siglas pueden producir derivados (como de "sida", "sidoso") y plurales como "TALGO", "talgos". En otras ocasiones, las siglas pasan a escribirse en minúscula, después de haber pasado por una fase de mayúsculas: OVNI > ovni, SIDA > sida.

La consideración de una sigla determinada como un nombre más, con olvido de su peculiar origen y significado, es aún más patente en los préstamos. Así, por ejemplo, en Fiat, radar ${ }^{18}$, láser, Simca, delco, etc. En estos casos, al estar formada la sigla sobre una secuencia sintagmática perteneciente a una lengua distinta a la española, la reconstrucción de su base precisa la competencia de la lengua a que esta pertenece ${ }^{19}$.

Puede decirse que una sigla se halla tanto mejor integrada como elemento del léxico, cuanto más borrada está su motivación original. Nos encontramos entonces con un verdadero nuevo lexema en la lengua. Y, una vez que la sigla funciona de hecho

\footnotetext{
${ }^{16}$ Seco, M. (1977) El léxico de hoy. En: Comunicación y lenguaje, p. 193.

${ }^{17}$ www.fundeu.es

${ }^{18}$ Radar, se ha convertido, en Inglés, en primer término de una familia de derivados de siglas, terminadas todas en -ar: colidar, hipar, sodar, sofar y sonar.

${ }^{19}$ Las siglas en las que se ha producido el paso de nombre propio a común han sufrido un proceso semántico de generalización. Las siglas en las que se ha producido el paso de nombre propio a común han sufrido un proceso semántico de generalización.
} 
como un nombre, entra necesariamente en el ciclo de transformaciones inherentes a cada palabra base ${ }^{20}$, es decir, se constituye en paradigma derivativo. Por tanto, la creación de los derivados a partir de una determinada sigla es signo inequívoco del nuevo estatuto lingüístico que esa sigla ha adquirido en la conciencia de los hablantes.

La pronunciación silábica es la que lee la sigla como una palabra: JASP, [jasp]. Esta última surge automáticamente siempre que el hablante sea capaz de pronunciarla según la fonética de su lengua, lo que produce una "sensación de familiaridad". Por ello, procurando que el hablante se sienta cómodo, se crean los sigloides, que integran grafemas con el objetivo de facilitar la pronunciación y evitar el deletreo, deformando el concepto de sigla. La pronunciación de la sigla indica su mayor lexicalización, su mayor incorporación al léxico habitual, mayor en las siglas pronunciadas (que la lingüística tradicional llamaba acrónimos), después en las deletreadas. Por ello, en bastantes casos la extrañeza del significado de una sigla hace que convenga desarrollarla para que el receptor entienda el mensaje; por ejemplo, pocos comprenderían el significado de las siglas PUR, que significa Partido Ucraniano Revolucionario.

En cuanto a la formación del plural, en la lengua oral suelen formar el plural como otras palabras: de ONG, /oenegés/; de DVD, /deuvedés/; de PC, /pecés/; de UCI, /ucis/. En la lengua escrita, las normas de las Academias de la Lengua establecen que carecen de plural (no hay que escribir -s o -es al final) y que el número ha de expresarse añadiendo un determinante a la sigla: varios PC, unas ONG. En el uso, el plural con -s, ya sea con o sin apóstrofo, está muy extendido; la Fundéu y ortotipógrafos como Xosé Castro recomiendan el empleo de una -s para que el plural prosódico y semántico tenga reflejo gráfico.

\subsection{El uso de los artículos en las siglas}

Existen determinados artículos de siglas establecidos en el léxico, como por ejemplo: A.M.P.A (Asociación de Madres y Padres de Alumnos) llevan el artículo determinado femenino LA por ser el sustantivo con el que concierta el artículo el sustantivo femenino Asociación, palabra femenina que comienza por A átona. En las siglas el artículo concuerda con el primer sustantivo de la sigla: La ONU (Organización de las Naciones Unidas), la OTAN (Organización del Tratado del Atlántico Norte), la UNESCO (Organización de las Naciones Unidas para la Educación, la Ciencia y la Cultura).

Las letras que forman siglas se escriben con mayúscula $\mathrm{y}$, por regla general, sin puntos (ISBN, ISSN), sobre todo cuando esas siglas han pasado a formar palabras, esto es, cuando constituyen acrónimos. La generalización de los acrónimos puede incluso permitir escribirlos con minúscula, total o parcialmente. Ejemplos: uvi, Mercosur. El plural de las siglas se construye haciendo variar las palabras que las

\footnotetext{
${ }^{20}$ Guillbert, L. (1975) La creativité lexicale. París: Larousse, p. 250.
} 
acompañan, como los TAC.

\subsection{El género y la acentuación de las siglas}

Las siglas adoptan el género de la palabra que constituye el núcleo de la expresión abreviada, que normalmente ocupa el primer lugar en la denominación: el FMI, por el «Fondo» Monetario Internacional; la OEA, por la «Organización» de Estados Americanos».

Las siglas son una excepción a la regla que obliga a utilizar la forma el del artículo cuando la palabra femenina que sigue comienza por / a/ tónica; así, se dice la AFE (y no el AFE), por «Asociación» de Futbolistas Españoles, ya que la palabra Asociación no comienza por /a/ tónica.

Las reglas que atañen a la escritura de los grafemas de una sigla son flexibles porque reflejan el avance de su proceso de incorporación al lenguaje habitual. Cuando se compone la sigla, aunque las iniciales de los términos originales lleven acento la sigla nunca la lleva, puesto que su pronunciación es nueva, y, por ello, también la sílaba tónica, al contrario que las abreviaturas. Tampoco se deben tildar las siglas que por reglas ortográficas deban tildarse, como CIA, que si fuera una palabra normal, para acentuarse la i debería ser tildada, pero en el caso de las siglas esto no es así21.

Además, el filólogo Leonardo Gómez Torrego afirma: “Las siglas son palabras formadas con las iniciales de otras palabras que constituyen un todo" 22 (Gómez Torrego, 2000). En opinión de este autor, las características más destacadas de las siglas son las siguientes:

- Las letras de las siglas se escriben con mayúscula y sin puntos, excepto en el caso de los acrónimos.

- Cuando la sigla va acompañada de un artículo, por lo general, este concuerda en género y número con la primera palabra de la sigla: el BOE (Boletín Oficial del Estado).

- Las siglas, por lo general, no tienen plural, pero este puede aparecer en las palabras que las acompañen: los CD-ROM. No obstante, se utilizan erróneamente muchas siglas en plural como: ONG's o APAs.

- Existen tres formas de pronunciar la siglas: deletreando cada inicial (ATS, [ateése]; realizando una lectura corriente (UNED, [unéd]; y mezclando las dos formas anteriores (CSIC, [cesíc]).

Sin embargo, ocurre con frecuencia que se confunden los significados de siglas y abreviaturas. En este sentido, es fácil decir dónde están las diferencias. Cuando las siglas se leen en voz alta, se pronuncian, generalmente, las letras o las sílabas que las

\footnotetext{
${ }^{21}$ Diccionario Panhispánico de Dudas (2005). Madrid: Santillana, p. 603.

${ }^{22}$ GÓMEZ TORREGO, L. (2000). Ortografía de uso del español actual. Madrid: SM.
} 
componen, en las abreviaturas, se lee la palabra entera y no las letras que componen la abreviatura: pza. se lee plaza, no pza ${ }^{23}$.

\subsection{Casos prácticos en la Red}

En los titulares de los espacios Web de los principales periódicos digitales nacionales se observa la inclusión de un considerable número de siglas. Por ejemplo:

Martes, 23 de agosto de 2011

El País: PSOE; IVA; OTAN; ONU; JMJ; BCE; EEUU; ETA; SGAE: FMI.

La Razón: CIU; PP; PSOE; PNV; IVA; OTAN; PSG; EEUU; BCE; IBEX; JMJ; ETA; SGAE; HP; PC.

ABC: OTAN; PP; PSOE; IVA; PNV; CIU; IU; INE; JMJ.

El Mundo: BCE; OTAN; CNT; SGAE; ONU; UE; AFE; JMJ; ETA; EEUU; BCE; FMI.

También, en el cuerpo de la información, se localizan las siglas con su correspondiente significado:

- Por su parte, el presidente de Convivencia Cívica Catalana (CCC), la entidad que apoya a las familias que recurrieron al Supremo, Francisco Caja, receló de una decisión que tildó de "irregular aunque legal" por el hecho de que haya sido de oficio y no a petición de parte, y que lamentó (LA RAZÓN, 16-9-2011).

- El rector de la Universidad de Castilla- La Mancha (UCLM), Ernesto Martínez Ataz, pidió "disculpas" a la presidenta de la Comunidad de Madrid, Esperanza Aguirre, que es criticada en un artículo periodístico que sirve de base para un examen de la Selectividad de los estudiantes castellanomanchegos (LA RAZÓN, 14-9-2011).

- El pronunciamiento de ayer a favor de la inmersión lingüística obligatoria, contra la que se había pronunciado el Tribunal Superior de Justicia de Cataluña (TSJC) (LA RAZÓN, 14-9-2011).

- Este órgano regulador se constituye, según reconoce la nota del Ministerio de Fomento, bajo lo previsto en la Ley de Economía Sostenible (LES), que fija un consejo de siete miembros (EL PAÍS, 27-8-2011).

- El Consejo Nacional de Transición (CNT) sabe muy bien que esta contienda no se acabará hasta que Gadafi aparezca, vivo o muerto (EL MUNDO, 26-8-2011).

- Las cuotas participativas de Caja Mediterráneo (CAM) costaban ayer a primera hora

${ }^{23}$ Diccionario de español urgente (2000). Madrid: SM, pp. 404-405. 
de la mañana 3,4 euros (EL MUNDO, 26-8-2011).

- Han hecho falta siete reuniones para que la Asociación de Futbolistas Españoles (AFE) y la Liga de Fútbol Profesional firmaran un pacto de honor para desconvocar la huelga que ya se cobró como víctima la primera jornada (ABC 26-8-2011).

- Entre las tres de la tarde del viernes y las ocho de la tarde de ayer, se produjeron once accidentes mortales, según datos de la Dirección General de Tráfico (DGT) (ABC 29-8-2011).

$$
\text { SIGLAS MÁS REPRESENTATIVAS EN ESPAÑA }
$$

\begin{tabular}{|c|c|}
\hline A E N A & $\begin{array}{c}\text { Aeropuertos Españoles y Navegación Aérea } \\
\text { A N E C A }\end{array}$ \\
\hline A V E & Alta Velocidad Española \\
\hline AVT & Asociación de Víctimas del Terrorismo \\
\hline B O E & Boletín Oficial del Estado \\
\hline C A M P S A & Compañía Arrendataria del monopolio de Petróleo \\
\hline C E P S A & Compañía Española de Petróleos, S. A. \\
\hline C G P J & Consejo General del Poder Judicial \\
\hline C N I & Centro Nacional de Inteligencia \\
\hline
\end{tabular}

\begin{tabular}{|c|c|}
\hline CO U & Curso de Orientación Universitaria \\
\hline C S I C & Consejo Superior de Investigaciones Científicas \\
\hline D R A E & Diccionario de la Real Academia Española de la Lengua \\
\hline E M T & Empresa Municipal de Transportes \\
\hline E S O & Educación Secundaria Obligatoria \\
\hline I G N & Instituto Geográfico Nacional \\
\hline I N A P & Instituto Nacional de Administración Pública \\
\hline I N E & Instituto Nacional de Estadística \\
\hline
\end{tabular}




\begin{tabular}{|c|c|}
\hline I N E M & Instituto Nacional de Empleo \\
\hline I N M & Instituto Nacional de Meteorología \\
\hline I V I M A & Instituto de la Vivienda de Madrid \\
\hline L O E & Ley Orgánica de Educación \\
\hline L O U & Ley Orgánica de Universidades \\
\hline MC U & Ministerio de Cultura \\
\hline M E C & Ministerio de Educacióny Ciencia \\
\hline MF O M & Ministerio de Fomento \\
\hline R A E & Real Academia Española de la Lengua \\
\hline R E N F E & Red Nacional de Ferrocarriles Españoles \\
\hline R N E & Radio Nacional de España \\
\hline
\end{tabular}

\begin{tabular}{|c|c|}
\hline RT V E & Radio Televisión Española \\
\hline U G T & Unión General de Trabajadores \\
\hline U N E D & Universidad Nacional de Educación a Distancia \\
\hline
\end{tabular}

\section{CONCLUSIONES}

Son varios los aspectos de la siglografía que se disputan la atención del lingüista, desde su materialización grafémica hasta los procesos de conversión de una determinada sigla en un lexema, base de múltiples derivaciones. En lo que respecta a la Lengua Española, sólo unos pocos han recibido alguna atención desde la perspectiva lingüística. No obstante, quedan pendientes de un detenido estudio cuestiones tan medulares como son las ortográficas, prosódicas y fonosintácticas.

Como ya se ha expresado, no se puede decir que el fenómeno de la sigla sea de reciente aparición, pero sí es patente su proliferación en el léxico castellano, sin gran diferencia de lo que está ocurriendo en todas las lenguas de cultura. Las siglas han invadido el vocabulario actual, con aceleración creciente, de tal manera que apenas hay ya esfera que no haya capitulado el empuje poderoso de este fenómeno lingüístico. Es muy numeroso el volumen de siglas que leemos en cualquier periódico o revista de actualidad, así como en tratados técnicos, científicos o humanísticos, sin olvidar su empuje mediante las Nuevas Tecnologías. Los periódicos digitales y páginas Web son otro reflejo de esta forma de escritura.

Si bien es cierto que en teoría puede prescindirse de los derivados de las siglas, en la práctica, el uso estilístico inclina a veces al escritor o al hablante a servirse de ellos; sobre todo, al escritor, dada la mayor frecuencia de empleo que alcanzan 
estas formaciones en la lengua escrita.

\section{BIBLIOGRAFÍA}

Alba De Diego, V. (1973) Marcas, abreviaciones y siglas en el lenguaje publicitario. En Prohemio, IV-3, pp. 349-378.

Alonso, D. (1962) La invasión de las siglas. En Del Siglo de Oro a este siglo de siglas, p. 7.

Almela, R. (1999) Procedimientos de formación de palabras en español. Barcelona: Ariel.

Alvar Ezquerra, M. \& Miró, A. (1983) Diccionario de siglas y abreviaturas. Madrid: Alhambra.

Casado Velarde, M. (1979) Creación léxica mediante siglas. En Revista Española de Lingüística, núm 9, Vol. 1, pp. 67-88.

Diccionario Panhispánico de Dudas (2005). Madrid: Santillana.

Diccionario de español urgente (2000). Madrid: SM.

Diego, G. (1968) Por las siglas de las siglas. En La Gaceta Literaria, p. 40.

Figueroa, L. (1969) Algunas observaciones sobre siglas usuales en Bogotá. En Thesaurus. BICC, XXIV.

Gómez Torrego, L. (2000) Ortografía de uso del español actual. Madrid: SM.

Guilbert, L. (1975) La créativité lexicale. París: Larousse.

Lang, M. F. (1997) Formación de palabras en español. Madrid: Cátedra.

Lapesa, R. (1963) La lengua desde hace cuarenta años. En Revista de Occidente, núm. 8-9, p. 201.

Martín Camacho, J. C. (2008) Sinonimia y polisemia en el léxico científico. El caso de las abreviaturas, las siglas y los epónimos. En Olza, I., Casado, M. \& González, R. (ed.) Actas del XXXVII Simposio Internacional de la Sociedad Española de Lingüística (SEL). Pamplona: Servicio de Publicaciones de la Universidad de Navarra.

Miranda, J. A. (1994) La formación de palabras en español. Salamanca: Colegio de España.

Rodríguez González, F. (1993) Las siglas como procedimiento lexicogenésico. En Estudios de Lingüística.( 9), 9-24. 
Rosell, A. (1967) Escritura de formas siglares. Montevideo: La Paz.

Rosenblat, A. (1967) El futuro de la lengua. En Revista de Occidente, núm. 56-57, p.164.

Seco, R. (1988) Manual de Gramática Española. Madrid: Aguilar.

Seco, M. (1977) El léxico de hoy. En Comunicación y lenguaje, pp. 181-202.

\section{Carlos Oliva Marañón}

Doctor en Ciencias de la Comunicación. Profesor de la Facultad de Ciencias de la Comunicación de la Universidad Rey Juan Carlos en las áreas de Periodismo, Comunicación Audiovisual y Magisterio. Investigador del Proyecto La Lengua de los textos multimedia: e-learning, financiado por la Comunidad de Madrid y la Universidad Rey Juan Carlos (Ref. URJC-CM-2010-CSH-5585). Ha trabajado como Investigador en los Proyectos Un nuevo léxico en la Red, La calidad del español en la Red y Memorias de Investigación del Consejo Superior de Deportes. 